\title{
Exploration and Practice of Practice Course Teaching Model Based on PBL Teaching Model
}

\author{
Dong Aoshuang*, Gao Tianha \\ Software College, Northeastern University, Shenyang, China \\ Email address: \\ dongas@swc.neu.edu.cn (Dong Aoshuang), gaoth@swc.neu.edu.cn (Gao Tianhan) \\ ${ }^{*}$ Corresponding author
}

\section{To cite this article:}

Dong Aoshuang, Gao Tianha. Exploration and Practice of Practice Course Teaching Model Based on PBL Teaching Model. Science Innovation. Vol. 7, No. 4, 2019, pp. 129-132. doi: 10.11648/j.si.20190704.15

Received: October 21, 2019; Accepted: November 14, 2019; Published: November 18, 2019

\begin{abstract}
This paper analyses the current situation and problems of university education at the present stage, and puts forward that in practical courses, we should make full use of the achievements of teachers' research practice and the training mechanism of university and enterprise cooperation, integrate PBL teaching mode with traditional practice process, design project topics by a team of teachers with practical experience and practical background, and organize students to follow. Teachers can inspire and promote students' learning process in the course, and formulate reasonable evaluation methods. Introducing PBL teaching mode into practice course can improve students' innovative ability. This paper elaborates in detail from five aspects: teaching method formulation, project design, implementation process, evaluation management and learning effect summary.
\end{abstract}

Keywords: Problem-Based Learning Teaching Model, Innovative Ability Training, Practical Courses

\section{基于PBL教学模式的实践课程教学改革的探索与实践}

\author{
董傲霜", 高天寒 \\ 东北大学软件学院, 沈阳, 中国
}

\section{邮箱}

dongas@swc.neu.edu.cn（董傲霜）, gaoth@swc.neu.edu.cn（高天寒）

\begin{abstract}
摘要: 分析现阶段大学教育的现状和问题, 提出在实践类课程中充分利用教师科研实践的成果和校企产学研合作培养 机制, 将PBL教学模式与传统的实践过程进行融合, 由具有实践经验和实践背景的教师团队设计项目题目, 组织学生 按照小组的方式主动进行学习, 教师在课程过程中启发、推动学生的学习过程, 并制定合理的评价方式。引入PBL教 学模式的实践课程可以提高学生的创新能力, 文章从教学方法制定、项目的设计、实施过程、评价管理和学习效果总 结五个方面进行具体阐述。
\end{abstract}

关键词: PBL教学模式, 创新能力培养, 实践课程

\section{1. 引言}

基于问题的学习(Problem based Learning)于 20世纪中 期起源于加拿大麦克马斯特大学 [1]。该学习过程把学习者 放置在复杂的有实际意义的问题情境中, 引导学习者主动
参与并自己寻找问题答案的教学模式[2]。PBL教学中教师 提出真实且较为复杂的问题, 学生组成团队参与学习, 在 学习过程中调研并分享各自学习成果, 创造性地完成学习 的任务, 教师对学习过程进行检查和评估, 以确定是否达 到学习的目的, 并通过反思修正教学的策略[3]。通过PBL 
学习培养学生观察和思考的能力, 分析解决问题的能力, 归纳总结和沟通的能力。PBL教学模式给创新人才培养的 的目标提供了一个很好地解决方案 [4-5]。

软件技术的飞速发展使其在经济发展中起着越来越 重要的作用。在各个应用领域中的应用要求软件的从业者 不但要具有软件基础知识、基础理论和实践动手能力, 更 要求从业者在所从事的军事、生产、经济等领域有一定的 知识背景和经验, 同时是否具有创新能力也成为对其评价 的一个重要指标[6-7]。PBL教学模式强调以学生的主动学 习为主, 把学习过程设置在一个问题领域中, 在教师的积 极指导下偏重小组合作学习和自主学习, 通过提出问题、 建立假设、手机资料、论证和总结[8]。让学生合作解决真 实问题, 学习相关知识, 培养学生的自主学习能力, 提高 学生的分析问题解决问题的能力[9-10]。

软件学院的软件工程相关专业把实用性、复合型、国 际化作为学生的培养目标, 在培养计划中致力于培养具有 扎实基础、专业特长鲜明、知识结构合理、适应性强的复 合型人才, 要想得到这样的效果需要在课程中引入应用背 景领域的相关知识的学习, 这样可以提高学生的专业技能 和实践项目能力, 积累相关行业的经验和背景知识, 提升 学生的实践创新能力 [11]。借鉴PBL教学模式在国内外大 学课程中的成功经验, 在软件学院的专业实践类课程中引 入PBL的教学改革, 是实现专业培养目标、实现创新型人 才培养的一种新途径[12-13]。

\section{2. 研究背景分析}

目前国内大部分软件工程专业的实践课程采用的教 学方式依然是按照教师制定的实践内容完成一种题目的 设计和实现过程。学生在实践过程中完成的课题知识为了 完成对于相关知识点的实践能力的学习与掌握, 学生在学 习过程中被动, 题目大多和实际的应用场景距离较远所以 很难激发学生的兴趣, 所以学生在学习过程的主动性和积 极性较差, 题目的实现也不会让学生具有成就感, 这都很 难激发学生的创新能力。

在软件学院的软件工程、信息安全和数字媒体的专业 的培养过程中引入PBL教学理念, 以学生为中心, 以“专 业综合实训”、“企业项目实训”等适合引入PBL的实践课程 教学作为过程, 以学院成立的工程中心为依托, 在工程中 心的教师团队和相关企业专业的组织下共同完成对于大
数据、人工智能、计算机金融、信息安全和虚拟现实等领 域的知识、技术的学习, 可以激发学生的学习兴趣, 积累 学生的项目经验, 培养学生的创新能力。这不仅可以有效 促进教学体制的连贯性, 还可以进一步完善与前沿技术相 关的课程体系。采取PBL教学模式, 可以在提升学生上述 基本素质的同时有效地培养其专业技能与实际项目参与 能力, 提高学生的创新能力[14-16]。

\section{3. 教学方法的制定}

在培养计划中选择具有一定时段的综合性实践课程 引入PBL教学模式。“专业综合实践”、“企业项目实训” 是本科生培养计划第二学年、第三学年夏季学期的两个综 合性实践课程, 在课程中开展实际项目开发训练, 让学生 体验真实的企业开发环境, 感受真实的软件开发“实战”过 程, 培养学生的职业素养, 有效提升学生的分析与解决问 题能力、交流与组织协调能力、主动获取知识能力、科学 创新能力、工程实践能力与国际竞争能力, 从而实现理论 知识与专业技能相互提升的实训效果, 提高学生就业竞争 力。

软件学院在三个专业的“专业综合实践”、“企业项目 实训”课程中引入PBL教学理念, 在学习过程中以学生为 中心, 让学生组成学习小组, 由学院的工程中心的教师团 队组织设计课程的教学目标, 按照教学目标设计确定问题 的情景, 由教师按照问题情景提供设计题目或者学生小组 自拟题目。在整个课程中小组成员按照问题所需要掌握的 知识点进行学习、设计、论证和评估, 教师组织对于团队 的问题分析、学习的讨论, 推动形成问题的解决方案, 并 组织在小组间每周至少一次进行分享和互相学习。除此之 外教师还要组织对于学习过程的评估和反馈, 对学生的学 习过程和学习成果进行评估和反馈。整个教学过程中突出 以解决问题为主线, 突出学生的自主学习, 督促学生的自 我反省、不断进步, 提高学生的学习能力和分析问题和解 决问题的能力。通过不同范围的讨论、分享、评价, 激发 学生的学习兴趣, 激发学生的学习兴趣, 培养学生的创新 能力。

教学改革以PBL教学团队建设、项目制定、团队管理 方法、学习过程管理方法、评价方法制定的研究为PBL教 学改革的实施路线。按照PBL的实施过程设计了 PBL教学 改革在实践课程中的实施方式, 具体的流程如图1所示。

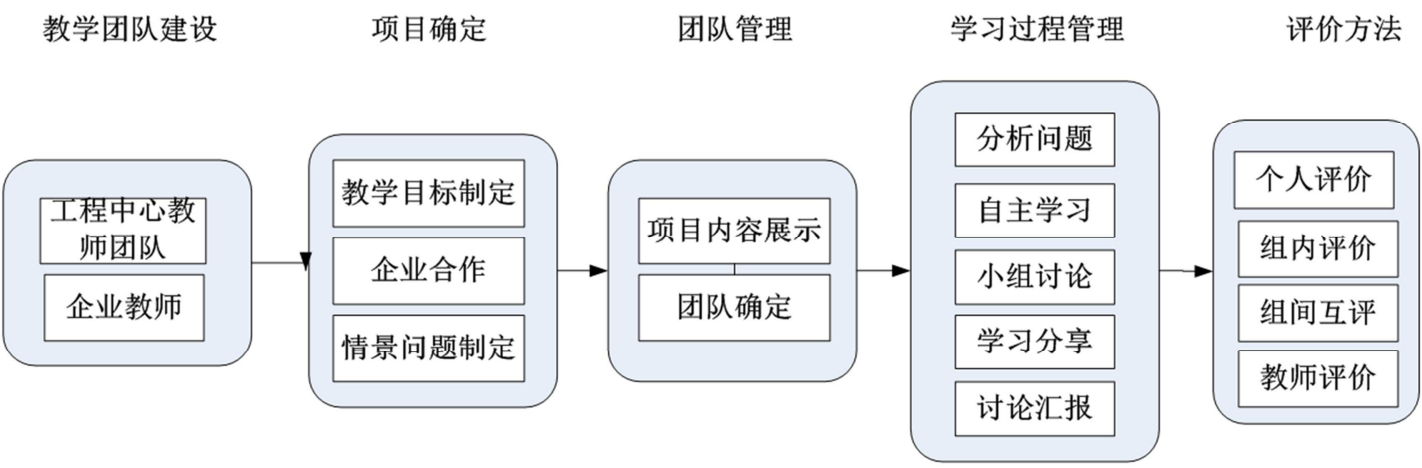

图1 教学改革实施路线图。 


\section{4. 项目的设计}

首先课程先依托学院成立的工程中心的教师组成 PBL教学改革教师团队，同时吸纳合作企业的业内人士， 承担对于教学目标的制定、情景问题的制定。根据工程中 心和相关企业的特点, 确定对于实践课程的教学目标, 提 出对于“专业综合实践”、“企业项目实训”课程的教学目标 和要求, 通过实践课程的学习学生应具备的自主学习能 力、分析问题解决问题能力的要求。并由这个教学目标分 解成在“专业综合实践”、“企业项目实训”课程学习中的学 习过程。

项目团队教师须以高度责任感及勇于开拓创新的精 神投入课程改革工作, 能结合自己所做的科研课题和企业 调研的结果提出与时俱进的教学要求。同时在对于教学目 标确定和情景问题制定的过程中既要考虑专业培养计划 的要求, 又要考虑行业领域对人才的能力、知识背景的要 求, 确定与行业发展相适应的教学目标、情景问题。

结合实践课程在专业教学中的地位和要求, 按照开设 的学期和开设的时间要求, 首先教学改革教师团队首先要 确定教学的目标, 明确在课程教学过程中各个阶段对学生 自主学习目标的要求, 分析问题和解决实际工程问题的能 力的要求。

例如软件学院的数字媒体专业在对“企业项目实训” 的教学目标确定后, 结合泛虚拟现实技术的特点, 将虚拟
现实教学的内容划分成三维物体的表示、三维软件开发设 计原理、三维交互设备原理和三维交互设计等知识模块, 重点培养学生对于VR、AR项目研发的分析问题和解决问 题的能力。结合对于知识学习和能力培养的要求确定问题 情景主要为旅游、教育、医疗、工业控制、移动互联网和 局势应用等为典型的应用场景进行设计问题。设计的问题 可与行业内有经验的开发者进行探讨细化, 对相关教学内 容和能力的要求进行相关性分析, 保证教学内容和目标的 实施。

在教学改革过程中教学团队要总结出相关活动的经 验, 对教学目标确定、情景问题的提出和项目的确定过程 中的方式、方法、原则等形成可供参考的流程和细则。

\section{5. 实施过程}

在确定项目内容之后学生组成小组进行团队的学习 过程。由于国内学校学习条件的限制, 如何保证才队能利 用有限的条件（供电时间有限、空间利用时间有限）下进 行充分、有效、安全地学习, 需要团队提出一套适合国情 的管理方案。

在数字媒体专业的引入PBL教学模式的“企业项目实 训”课程中, 给出了的部分题目如表1所示。

表1 项目名称表。

\begin{tabular}{ll}
\hline 项目名称 & 名称 \\
\hline $\mathrm{AR}$ 汽车展厅 & 在汽车展厅中通过AR眼镜识别展厅中的汽车样车车型, 用户可以查看汽车参数、汽车换色交互等操作。 \\
$\mathrm{AR}$ 交警 & 通过AR眼镜识别违章停靠的汽车车牌, 实现交警的移动执法。 \\
$\mathrm{AR}$ 智能仓储 & 通过AR眼镜识别仓储中的存放的零件的二维码信息对仓储进行智能盘点。 \\
虚拟展播室 & 实现某一特定内容的虚拟教学过程, 如摄影课程中光圈、速度和快门调节的效果教学过程。 \\
虚拟医疗卫生检验检疫教学 & 实现基于WEB的一个教学系统, 演示特定检验检疫所用设备、检验过程的教学。 \\
$\mathrm{AR}$ 人脸识别 & 实现通过AR眼镜进行人脸识别和简单交互的系统。 \\
$\mathrm{AR}$ 展馆 & 通过AR眼镜实现一个展馆并完成相关视频、动画等交互过程。 \\
\hline
\end{tabular}

引入PBL教学模式的实践课程要求学生以小组的形 式进行项目化的学习, 小组人数限定在4-6人之间。在一 个开放性实验室中为每个项目小组分配项目学习的环境, 包括桌椅、电源、上网环境、讨论用白板、打印设备、AR 交互设备等, 小组进行自我管理, 首先进行问题的分析, 结合分析的结果进行相关问题的学习和研究, 指定项目实 施的分工和计划，并将分工计划上报指导教师并进行组间 讨论。小组成员可以按照分工结合工程中心提供的项目案 例库进行学习, 也可以结合教师的课件、网上的慕课、案 例进行学习。教师通过定期每周至少 1-2次听取小组汇报, 每周1次听取组间学习内容分享、旁听学生讨论形式对学 生的学习进度和学习过程进行控制, 启发学生的思维, 激 发学生的学习兴趣, 保证学生自我学习的实现, 也可以防 止学生疏于管理。

\section{6. 评价管理}

学生在课程学习过程的评价从多个方面获取, 首先学 生在学习过程中可以对自己从项目学习的过程中获取到
的知识和能力的提高进行个人评价, 小组内部成员之间可 以在项目学习的过程中指定组内成员之间的评价, 通过组 间学习的分享可以进行小组之间的评价, 教师可以根据项 目消息过程中学生在小组中的作用和表现进行评价。多元 化的评价体系可以从学生的知识背景、学习能力、应变能 力、团队合作能力、讨论过程、项目展示过程等多个角度 多个方面进行评价, 可以鼓励学生个性化发展和创新能力 的培养。

在数字媒体专业的引入PBL教学模式的“企业项目实 训”课程中, 每周一次的组间分享均进行了互评和教师评 价, 在课程结束的答辩过程中进行了组内评价和教师评 价。最后成绩比例如表2所示。

表2 评分表。

\begin{tabular}{lll}
\hline 评价项目 & 占比 & 备注 \\
\hline 文档撰写 & $30 \%$ & 每个小组一份文档, 小组成绩也是个人成绩 \\
组间互评 & $20 \%$ & 按小组排序给出成绩 \\
教师评价 & $40 \%$ & 按照学生每周评价和最终答辩评价给出成绩 \\
组内互评 & $10 \%$ & 小组内成员按照排序给出成绩 \\
\hline
\end{tabular}




\section{7. 学习效果总结}

在软件学院的相关专业的“综合专业实践”、“企业项 目实训”两个实践课程中引入PBL教学模式, 由具有一定 工程背景的教师团队按照技术发展的特点和专业培养方 向确定项目的内容, 由于项目多是来自企业实际的需求或 者具有一定前瞻性的研究课题, 所以学生在实践过程中兴 趣很高。学生按照自己的兴趣点进行选题, 自己组织和管 理设计和开发的过程。在项目的收集阶段大家分别去研究 和学习相关领域知识, 在工程中心提供的案例库中进行学 习研究, 并在组内、组间进行分享, 深入的研究和分析为 得到准确可行的设计方案提供了保障。组内的讨论、组间 的分享锻炼了学生的表达沟通能力, 也激发了学生的探索 创新的兴趣。在课程结束之后学生们都表示得到了很多的 锻炼, 并有一部分学生拿自己的设计作品继续参加相关设 计大赛和创新项目的研究。我院在相关实践课程中所做的 PBL教学改革活动, 对原有的实用性、复合型人才的培养 方向进行了深入细致的工作，取得了良好的效果。

PBL教学模式可以系统地完成实践课程的培养目标, 提高了学生学习的主动性, 提高了学生的创新能力。但由 于PBL教学模式和传统的授课方式不同, 所以在实际实施 中需要以下问题:

(1) 教学硬件资源：由于学生是以团队形式参与学 习, 自学、讨论、分享的活动在学习过程中占很大比例, 所以应为团队提供独立的学习空间、学习资源、设备资源。

（2）师资团队：承担PBL教学改革的教师应具有丰 富的理论知识、领域知识和工程经验, 最好能聘请具有教 学经验的企业业内人士参与, 所以师资团队的建设是进行 教学改革的关键。

(3) 学习过程安排: PBL学习需要学生投入更多的 时间, 所以在教学进程的安排一定考虑学生在学习上的投 入时间。

（4）评价体系: 制定出一套切实可行的完整的评价 体系可以调动学生的积极性, 要用评价鼓励学生参与学 习, 充分调动学生的积极性。

\section{参考文献}

[1] Khoo H E, Chhem R K, Problem-based learning: Issues and challenges $[\mathrm{J}]$. Annals of the Academy of Medicine Singapore, 2001, 30 (4): 338-339.
[2] 杜翔云, 科莫斯, 基于问题的学习: 理论与实践 $[\mathrm{M}]$. 高等 教育出版社, 2013.

[3] 刘莉, 惠晓丽, 胡志芬. 基于PBL理论的工科人才培 养途径探究 [J]. 高等工程教育研究, 2011(3).

[4] 刘春城, PBL教学模式在工程训练教学中的探索与实践.实 验技术与管理,2012,29(4):158-161.

[5] 李阳，侯阿临，张丽娟，利用PBL模式培养地方工科 院校创新型人才的研究 $[\mathrm{J}]$. 软件导刊. 教育技术, 2018(8):21-23.

[6] 冯晅, 梁文婧, 刘财,应用PBL教学方法培养学生自主学习 能力与创新思维一以地震勘探系列课程为例. 教育教学 论,2019,17:51-52.

[7] 沈艳霞, 赵芝璞, 王子斑, 基于项目的教学模式探索及其 实施.教育教学论,2019,17:165-166.

[8] 张文婧, 刘振宇, 李业荣, 基于混合式教学及雨课堂在大 学计算机基础中的应用与思考.教育教学论,2019,24:184-85.

[9] 郭小波, 刘永平,基于PBL教学模式的数据结构课程改革初 探.电脑知识与技术, 2019,15.11:122-25.

[10] 王淑勤, 武金锦, 陈司晗, PBL模式提高学生工程实践及创 新能力的应用.山东化工,2019,48.9:214-15.

[11] 吴许俊,姜枫,王巍,基于PBL与项目驱动的计算机网络课程 改革探索.计算机教育,2016,11:69-72.

[12] 佟延秋,基于PBL的软件工程课程实习模式研究.计算机教 育, 2015,12: 69-73.

[13] 曾志,周永福,融入PBL项目教学法的应用型软件人才培养. 计算机教育,2015,13:79-82.

[14] 蔡朝朝, 谢岚,李湘,案例法结合PBL教学模式在数据库课程 中的应用.计算机教育,2014,17:66-70.

[15] 杜秋霞,计算机教学中如何培养学生的创新能力.中国科技 博览,2012,30: 215.

[16] 王雅杰,基于问题的学习（PBL）在“多媒体技术与应用”实 践教学中的应用.实验技术与管理,2015,32.10:188-92. 